\title{
Pneumatic Compression Devices in Prevention of Intradialytic Hypotension
}

\author{
Minh Huan Dang*, Christopher Sia, Shane Fernando, Michael Phan, and Kim Wong \\ Department of Nephrology, Frankston Hospital, Australia
}

*Corresponding author: Minh Huan Dang, Department of Nephrology, 2 Hastings Road, Frankston, Victoria 3199, Australia, Tel: + 61 423933043, E-mail: huandg341@gmail.com

Received: 02 Nov, 2019 | Accepted: 11 Dec, 2019 | Published: 17 Dec, 2019

Citation: Dang MH, Sia C, Fernando S, Phan M, Wong K (2019) Pneumatic Compression Devices in Prevention of Intradialytic Hypotension. Int J Nephrol Kidney Fail 5(4): dx.doi.org/10.16966/2380-5498.187

Copyright: (C) 2019 Dang MH, et al. This is an open-access article distributed under the terms of the Creative Commons Attribution License, which permits unrestricted use, distribution, and reproduction in any medium, provided the original author and source are credited.

\begin{abstract}
Background: Intradialytic Hypotension (IDH) is a common clinical problem during Hemodialysis (HD) and is associated with increased mortality and morbidity. Pneumatic Compression Devices (PCD) are known to increase blood flow and venous return in the lower limbs. In patients on hemodialysis, PCDs may prevent IDH by reducing venous stasis and increasing central blood volume.

Methods: The study is a two-period, eighteen-treatment, randomized crossover trial. Patients at high risk of IDH were recruited. Patients underwent eighteen HD sessions, randomized to begin the first nine sessions either with or without PCDs. The primary outcome was the incidence of IDH, defined as an intradialytic decrease in Systolic Blood Pressure (SBP) from pre-dialysis SBP by at least $20 \mathrm{mmHg}$. Secondary outcomes were IDH with patient symptoms requiring intervention, nadir SBP, absolute maximum decrease in SBP, nadir SBP less than $100 \mathrm{mmHg}$, nadir SBP less than 90 $\mathrm{mmHg}$, net ultrafiltration and comfort on dialysis.
\end{abstract}

Results: 16 patients were recruited and randomized into 2 groups. There was no statistically significant difference in the incidence of IDH for PCDs and control $(58 \%$ versus $52 \%$, P-value $=0.31$ ). All secondary outcomes were not statistically significantly different between two arms (all P-values $>0.05)$. However, most patients reported improved comfort and better quality of HD sessions with application of PCDs.

Conclusions: PCDs did not reduce intradialytic hypotension. However, PCDs improved patient comfort during HD sessions.

Keywords: Hemodialysis; Pneumatic compression devices; Intradialytic hypotension; Comfort

\section{Introduction}

Intradialytic hypotension (IDH) is a common clinical problem occurring in 20-30 percent of dialysis sessions [1]. It is associated with increased mortality, hospitalization and cardiovascular complications including left ventricular hypertrophy and myocardial stunning [1-4].

The pathophysiology of IDH is complicated and poorly understood. It likely relates in part to a decrease in plasma volume associated with ultrafiltration [5]. Ultrafiltration is associated with a 6-10\% decrease in blood volume $[5,6]$ however despite this, Central Blood Volume (CBV) is preferentially maintained [5-7]. This predominantly occurs through vascular refilling from the lower limbs [5-7].

Pneumatic compression devices (PCD) are commonly used for the prevention of deep vein thrombosis [8]. Intermittent pneumatic compression has been demonstrated to increase velocity [9] and blood flow volume (up to $80 \%$ ) through the common femoral vein [10]. Similarly, lower limb compression bandages have been shown to decrease orthostatic systolic blood pressure decrements and symptoms of orthostatic hypotension in elderly patients with postural hypotension [11].

Studies of PCDs and their effect on hemodynamic parameters in dialysis patients are limited. Small case series have demonstrated improvements in muscle cramps, ultrafiltration and IDH [12-14]. A crossover trial evaluating the effect of PCDs in hemodialysis patients showed a significantly increased total body water reduction, suggesting a potential benefit in this population. The PCDs did not demonstrate a reduction in the decrement of CBV [15]. However, the study did not specifically recruit patients at high risk for IDH or volume overload and was underpowered to detect a decrease in IDH.

Our aim therefore was to evaluate the effect of PCDs on the incidence of IDH in a high-risk dialysis cohort.

\section{Methodology}

The study was a two-period, eighteen-treatment, randomized crossover trial. Written informed consent was obtained from all participants during recruitment period. The study protocol was approved by Peninsula Health Human Research Ethics Committee. The study was registered at Australian New Zealand Clinical Trial Registry (anzctr.org.au, ACTRN12616000524493).

\section{Study population}

Eligible participants were recruited from our outpatient hemodialysis unit at Frankston Hospital in Melbourne, Victoria, Australia. Frankston Hospital is a 454-bed public hospital located 
in the outer metropolitan area of the city of Melbourne and provides secondary and tertiary medical services. Eligible participants were greater than 18 years of age, had been on hemodialysis for at least 3 months, had capacity to provide informed consent and had intradialytic hypotension in at least $30 \%$ of hemodialysis sessions during the four-week period prior to recruitment. Exclusion criteria included recent unplanned, acute hospital admissions within the last 2 weeks, critical lower limb ischemia, pressure areas, known current deep venous thrombosis or pulmonary embolism, compartment syndrome, vascular access dysfunction, inability to provide informed consent and previous lower limb amputation.

\section{Study protocol}

Participants were randomized by a computer-generated number into two groups to start with either nine sessions with PCD or nine sessions without PCD (Figure 1). Group A participants started with nine hemodialysis sessions with PCD, follow by a one-week washout period, followed by nine hemodialysis sessions without PCD. Group B participants started with nine hemodialysis sessions without PCD, followed by nine sessions with PCD. There were no differences between treatment period and control period, except for

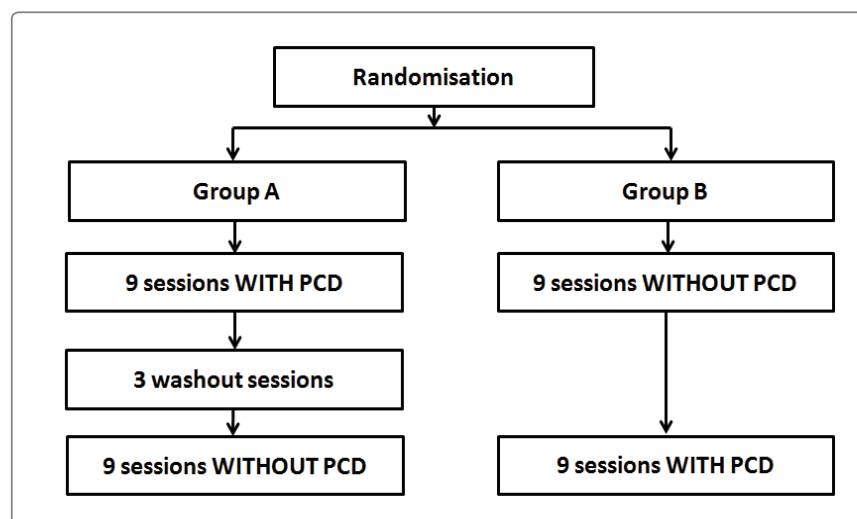

Figure 1: Study randomization and design. the application of PCD. Standard of care during hemodialysis was maintained throughout the duration of the study. Where possible, no changes were made to dialysis prescription parameters such as sodium dialysate and ideal body weight. Participants were advised to maintain their current antihypertensive medications during the trial.

During the intervention period, a Kendall Sequential Compression Device 700 Series was applied prior to commencement of hemodialysis and removed prior to patient's discharge from the unit. The device consists of a controller, non-disposable tubing and single-patient use compression sleeves. During the intervention sessions, the compression sleeve was applied to participants' legs. A pump performed intermittent compression during the duration of the dialysis. Each cycle of compression lasted 60 seconds. The compression sleeve comprised of 3 chambers. The lowest chamber, middle chamber and highest chamber applied sequential compression of $45 \mathrm{mmHg}$, $40 \mathrm{mmHg}$ and $30 \mathrm{mmHg}$, respectively. The PCD had a venous refill detection system, which optimized time between compressions, ranging from 20 to 60 seconds, based on the individual patients' venous refill time.

\section{Study outcomes}

Participant baseline characteristics were extracted from hemodialysis records and Frankston hospital medical database, with informed consent from all participants.

Weight, systolic blood pressure, diastolic blood pressure and heart rate were measured before the dialysis session prior to application of PCD. Intradialytic hemodynamic parameters were measured at onehour intervals, including blood pressure, heart rate, symptoms of IDH (muscle cramps, headache, dizziness, diaphoresis, nausea, vomiting) and any nursing intervention performed. Blood pressure was measured using Welch Allyn Connex vital sign monitor with automated blood pressure cuffs by hemodialysis nurse. Post-hemodialysis weight, ultrafiltration volume, blood pressure and any side effects from PCD were recorded.

The primary outcome was the incidence of intradialytic hypotension, which was defined as the presence of a decrease in systolic blood pressure from pre-dialysis blood pressure of $20 \mathrm{mmHg}$ or more at

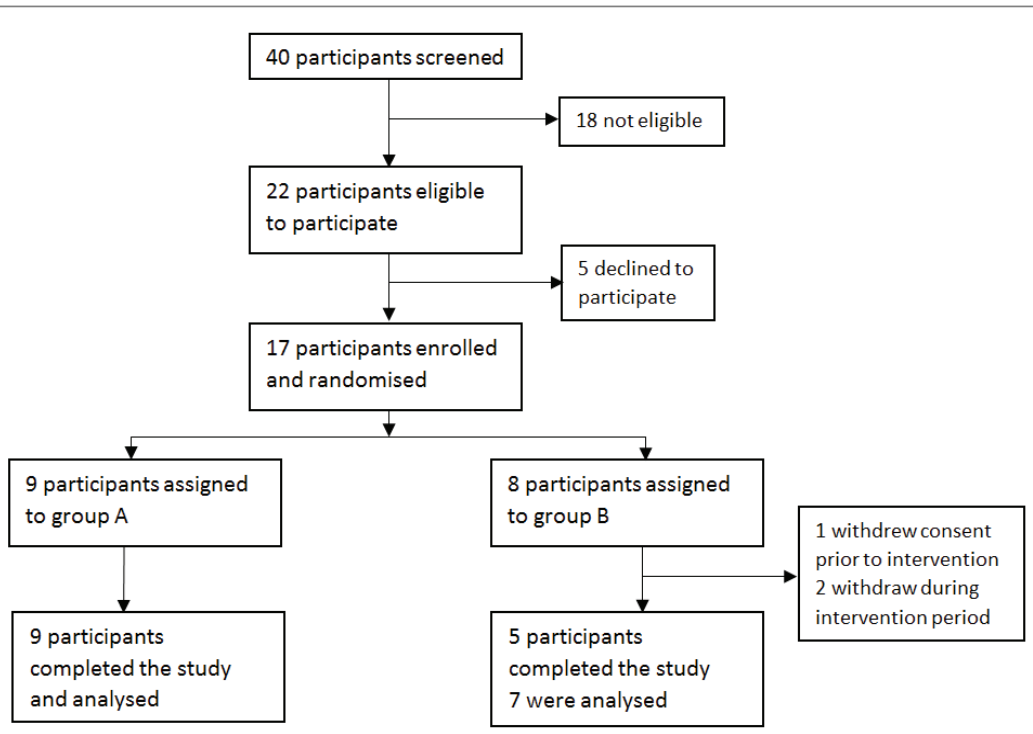

Figure 2: Study flow diaphragm. 
Table 1: Demographics data of participants.

\begin{tabular}{|l|c|}
\hline \multicolumn{2}{|c|}{ Overall (N=16) } \\
\hline Age median (years) (IQR) & $69.5(64.3-75.0)$ \\
\hline Male (\%) & $6(37.5)$ \\
\hline Time since initiation of dialysis (months) (IQR) & $88(21-117.75)$ \\
\hline Antihypertensive median (IQR) & $2(0-2)$ \\
\hline \multicolumn{2}{|c|}{ Cause of ESRF } \\
\hline Diabetes Mellitus (\%) & $10(62.5)$ \\
\hline Glomerulonephritis (\%) & $3(18.75)$ \\
\hline Obstructive uropathy (\%) & $1(6.25)$ \\
\hline Hypertension (\%) & $1(6.25)$ \\
\hline Other (\%) & $1(6.25)$ \\
\hline Charlson Comorbidity Index (IQR) & $7.5(5-8.5)$ \\
\hline & $11(68.75)$ \\
\hline Diabetes Mellitus (\%) & $11(68.75)$ \\
\hline Hypertension (\%) & $8(50)$ \\
\hline Left ventricular dysfunction (\%) & $3(18.75)$ \\
\hline Valvular heart disease (\%) &
\end{tabular}

Table 2: Study results.

\begin{tabular}{|l|c|c|c|}
\hline & $\begin{array}{c}\text { PCDs } \\
\text { (Intervention) }\end{array}$ & $\begin{array}{c}\text { No PCDs } \\
\text { (Control) }\end{array}$ & P Value \\
\hline $\begin{array}{l}\text { Intradialytic hypotension (SBP } \\
\text { drop by } 20 \mathrm{mmHg} \text { ) (\%) }\end{array}$ & 58.1 & 52.1 & 0.34 \\
\hline Nadir SBP (mmHg) mean (SD) & $120.1(19.5)$ & $119.7(19.3)$ & 0.85 \\
\hline Nadir DBP (mmHg) mean (SD) & $66.3(9.3)$ & $65.7(16.2)$ & 0.58 \\
\hline $\begin{array}{l}\text { Maximum drop in SBP (mmHg) } \\
\text { mean (SD) }\end{array}$ & $27.3(19.8)$ & $25.3(16.2)$ & 0.34 \\
\hline Nadir SBP <100 mmHg (\%) & 16.2 & 13.2 & 0.50 \\
\hline Nadir SBP <90 mmHg (\%) & 5.2 & 4.2 & 0.78 \\
\hline $\begin{array}{l}\text { SBP drop by } 20 \mathrm{mmHg} \text { with } \\
\text { symptoms and nursing } \\
\text { intervention (\%) }\end{array}$ & 7.4 & 8.3 & 0.83 \\
\hline Ultrafiltration (L) mean (SD) & $2.07(0.07)$ & $2.06(0.07)$ & 0.97 \\
\hline
\end{tabular}

Table 3: Adverse events.

\begin{tabular}{|l|c|}
\hline \multicolumn{1}{|c|}{ Adverse events } & Number of events (\%) \\
\hline Discomfort & $4(33.3 \%)$ \\
\hline Pain, ache & $3(25.0 \%)$ \\
\hline Tingling & $2(16.7 \%)$ \\
\hline Leg cramps & $2(16.7 \%)$ \\
\hline Itchiness & $1(8.3 \%)$ \\
\hline Total & 12 \\
\hline
\end{tabular}

any time during dialysis. Secondary outcomes were intradialytic hypotension with symptoms and requiring nursing intervention, nadir systolic blood pressure less than $90 \mathrm{mmHg}$, nadir systolic blood pressure, nadir diastolic blood pressure, absolute maximum drop in systolic blood pressure from pre-dialysis systolic blood pressure, ultrafiltration achieved per session and symptoms of intradialytic hypotension.

Post study, a survey evaluating whether PCDs improved the quality of hemodialysis was conducted. Side effects of PCDs were also recorded during every intervention session and analyzed.

\section{Statistical analysis}

Statistical analysis was performed using Stata v14 (StataCorp LP, Texas, USA). All continuous variables were tested for normality of distribution using the Shapiro-Wilk test. Those variables that failed this test were transformed (by cube root) to achieve normal distribution and the transformed data was also tested for skewness and kurtosis to ensure transformed data was suitable for further statistical analysis using parametric tests.

For continuous variables, the comparison of whether compressions were applied or not was conducted using a t-test. Categorical variables were tested using $\chi 2$-test or Fisher's Exact test. Further tests for interactions of factors such as session number, compression and order of compression on the outcome were examined using two-way repeated measures ANOVAs and multiple logistic regression analysis. All tested data achieved a power $\geq 0.8$ when $\alpha=0.05$ for each test, thus an accepted statistical difference was found and could safely reject the null hypothesis when $\mathrm{P}<0.05$.

\section{Results}

\section{Study participants}

Participants were recruited at Frankston Hospital Hemodialysis Unit from April 2016 to May 2016. Forty patients were screened, of which twenty-two participants were eligible to participate (Figure 2). Seventeen participants consented and were enrolled in the trial. Five patients declined to participate in the study. Participants were randomized into group A (nine participants) or group B (eight participants). After randomization, prior to intervention, one participant from group $B$ electively withdrew consent due to concerns regarding side effects. Sixteen participants (nine in group A and seven in group B) were included in the analysis.

Baseline characteristics of sixteen participants who received intervention are presented in table 1 . The median age was 69.5 years (IQR 10.75 years) and $37.5 \%$ of participants were male. Diabetes was the most common reason for ESRD (Table 1).

Two participants from group B electively withdraw from the study during the intervention period. The reason for withdrawal was calf discomfort during hemodialysis after the PCD was applied. However, their data was used in the final analysis.

\section{Study outcomes}

Overall, analysis of primary and secondary outcomes showed no statistically significant differences between control and intervention. The primary outcome, intradialytic hypotension, occurred in 79 out of a total of 136 hemodialysis sessions (58.1\%) in the intervention arm compared to 75 out of a total of 144 sessions in the control group (52.1\%) $(\mathrm{p}=0.31)$. There was no difference between the control arm and intervention arm in mean nadir SBP $(\mathrm{P}=0.85)$, mean nadir DBP $(\mathrm{P}=0.58)$, maximum intradialytic SBP drop $(\mathrm{P}=0.34)$, incidence of nadir SBP less than $100 \mathrm{mmHg}(\mathrm{P}=0.50)$ and incidence of nadir SBP less than $90 \mathrm{mmHg}(\mathrm{P}=0.78)$ (Table 2). Adverse side effects likely related to PCDs were recorded in 12 out of 280 hemodialysis sessions, with the most common complaint being discomfort (4 sessions) (Table 3).

In our follow-up survey after completion of the study period, thirteen out of sixteen participants either agreed or strongly agreed that PCDs improved the quality of hemodialysis in term of comfort. No significant side effects were reported; however two patients withdrew during the study due to discomfort associated with PCDs. 


\section{Discussion}

In our current study, PCDs did not have a significant effect on the incidence of IDH, other hemodynamic parameters or symptoms of intradialytic hypotension. The study was adequately powered for our statistical analysis.

We hypothesize those PCDs ineffectiveness at reducing IDH was related to the multifactorial pathophysiology of IDH. Changes in blood volume hemodynamics, while important are unlikely to fully explain its mechanism. Failure to increase peripheral vascular resistance may be an important contributing factor to the development of IDH [7]. This may be due to inappropriate activation of the cardiodepressor reflex, resulting in increased parasympathetic and decreased sympathetic nervous system activity [16]. Cardiovascular diseases, such as left ventricular hypertrophy and myocardial ischemia, can greatly increase risk of developing IDH [3]. In our cohort, cardiovascular disease was highly prevalent with $50 \%$ of participants having a history of LVH or congestive heart failure. Cardiovascular dysfunction combined with the hemodynamic stress of hemodialysis has been proposed to cause regional enteric ischemia [4]. This may lead to translocation of endotoxin from intestinal bacteria, systemic inflammation and hypotension, perpetuating a vicious cycle [4]. In addition, it has been proposed that myocardial stunning occurs in some patients undergoing hemodialysis, although the mechanism is poorly understood [17]. While PCDs attempt to counter changes in blood volume on dialysis, they do little to address the various other important factors involved in IDH, possibly explaining the negative result of this study.

PCDs were well tolerated; with $81 \%$ of patients agreeing that they improved noticed an improvement in the comfort of dialysis compared to control. This important finding should not be underestimated. Withdrawal of dialysis is a common cause of death in end-stage renal failure $[18,19]$, highlighting the importance of comfort and quality of life in this population. In dialysis patients, foot massage therapy has been shown to increase sleep quality [20], reduce fatigue, pain and muscle cramping $[21,22]$. These trials raise the possibility that PCDs could be used to improve the tolerability of dialysis and quality of life in dialysis patients in future studies.

Our study has various limitations. Firstly, while the number of dialysis sessions evaluated was substantial, the number of individual subjects was limited. Secondly, the crossover design of the study eliminated many potential confounders however as the intervention period was over 6-7 weeks, we cannot exclude the effect of time as a potential confounding factor. However, the effect of time should have been mitigated by the initial randomization of patients to start with or without IPCs. In addition, other factors such as medications might also be an important confounding factor. Thirdly, the technical adjustment of the IPCs, such as degree of tightness, was not standardized but instead was tailored to patients' comfort. This may have influenced the effect on venous return, which we did not measure. Fourthly, we did not have access to central blood volume measurements, which may be one of the key factors in explaining IDH, particularly as blood pressure may not reflect intravascular blood volume. Finally, while all efforts were made to make no adjustments to dialysis parameters, $63 \%$ of participants' ideal body weight was changed during study period. Although the majority of the changes in ideal body weight was equal or less than $1 \mathrm{~kg}$ from the original ideal body weight, this may have influenced the intradialytic hemodynamic parameters.
The strength of the current trial is that it was the first adequately power trial to evaluate the effect of IPCs on a high-risk cohort for ICH. We also used thigh high pneumatic compression devices with a venous refill detection system to optimize the frequency and effectiveness of compressions and maximize the volume of lower limb venous return.

\section{Conclusion}

The use of PCDs did not decrease the incidence of intradialytic hypotension or symptoms of intravascular volume depletion. PCDs were well tolerated and improved the quality of dialysis in the majority of patients.

\section{Fundings}

The pneumatic compression devices used in the study was provided by Covidien. This research received no other specific grant from any funding agency.

\section{Conflict of Interest Statement}

None declared.

\section{References}

1. Stefansson BV, Brunelli SM, Cabrera C, Rosenbaum D, Anum E, et al. (2014) Intradialytic hypotension and risk of cardiovascular disease. Clin J Am Soc Nephrol 9: 2124-2132.

2. Shoji T, Tsubakihara Y, Fujii M, Imai E (2004) Hemodialysis-associated hypotension as an independent risk factor for two-year mortality in hemodialysis patients. Kidney Int 66: 1212-1220.

3. Chao CT, Huang JW, Yen CJ (2015) Intradialytic hypotension and cardiac remodeling: a vicious cycle. Biomed Res Int 2015: 724147.

4. Burton JO, Jefferies HJ, Selby NM, McIntyre CW (2009) Hemodialysisinduced cardiac injury: determinants and associated outcomes. Clin J Am Soc Nephrol 4: 914-920.

5. Lindsay RM, Shulman T, Prakash S, Nesrallah G, Kiaii M (2003) Hemodynamic and volume changes during hemodialysis. Hemodial Int 7: 204-208.

6. Prakash S, Reddan D, Heidenheim AP, Kianfar C, Lindsay RM (2002) Central, peripheral, and other blood volume changes during hemodialysis. ASAIO J 48: 379-382.

7. Jain AK, Lindsay RM (2008) Intra and extra cellular fluid shifts during the inter dialytic period in conventional and daily hemodialysis patients. ASAIO J 54: 100-103.

8. Cayley WE Jr (2007) Preventing deep vein thrombosis in hospital inpatients. BMJ 335: 147-151.

9. Ricci MA, Fisk P, Knight S, Case T (1997) Hemodynamic evaluation of foot venous compression devices. J Vasc Surg 26: 803-808.

10. Lurie F, Awaya DJ, Kistner RL, Eklof B (2003) Hemodynamic effect of intermittent pneumatic compression and the position of the body. J Vasc Surg 37: 137-142.

11. Podoleanu C, Maggi R, Brignole M, Croci F, Incze A, et al. (2006) Lower limb and abdominal compression bandages prevent progressive orthostatic hypotension in elderly persons: a randomized singleblind controlled study. J Am Coll Cardiol 48: 1425-1432.

12. Ahsan M, Gupta M, Omar I, Frinak S, Gendjar S, et al. (2004) Prevention of hemodialysis-related muscle cramps by intradialytic use of sequential compression devices: a report of four cases. Hemodial Int 8: 283-286. 
13. Onuigbo MA (2010) Bilateral lower extremity sequential compression devices (SCDs): a novel approach to the management of intra-dialytic hypotension in the outpatient setting--report of a case series. Ren Fail 32: 32-35.

14. Beninson J, Levin NW, Santiago G, Paparao P, Bobola N (1974) Use of intermittent pneumatic compression in hemodialysis. Proc Clin Dia Transplant Forum 209-213.

15. Tai DJ, Ahmed SB, Palacios-Derflingher L, Hemmelgarn BR, MacRae JM, et al. (2013) Pneumatic compression devices during hemodialysis: a randomized crossover trial. Nephrol Dial Transplant 28: 982-990.

16. Barnas MG, Boer WH, Koomans HA (1999) Hemodynamic patterns and spectral analysis of heart rate variability during dialysis hypotension. J Am Soc Nephrol 10: 2577-2584.

17. Zuidema MY, Dellsperger KC (2012) Myocardial Stunning with Hemodialysis: Clinical Challenges of the Cardiorenal Patient. Cardiorenal Med 2: 125-133.
18. Birmele B, Francois M, Pengloan J, Français P, Testou D, et al. (2004) Death after withdrawal from dialysis: the most common cause of death in a French dialysis population. Nephrol Dial Transplant 19: 686-691.

19. Findlay MD, Donaldson K, Doyle A, Fox JG, Khan I, et al. (2016) Factors influencing withdrawal from dialysis: a national registry study. Nephrol Dial Transplant 31: 2041-2048.

20. Unal KS, Balci Akpinar R (2016) The effect of foot reflexology and back massage on hemodialysis patients' fatigue and sleep quality. Complement Ther Clin Pract 24: 139-144.

21. Mastnardo D, Lewis JM, Hall K, Sullivan CM, Cain K, et al. (2016) Intradialytic Massage for Leg Cramps Among Hemodialysis Patients: a Pilot Randomized Controlled Trial. Int J Ther Massage Bodywork 9: 3-8.

22. Ozdemir G, Ovayolu N, Ovayolu O (2013) The effect of reflexology applied on haemodialysis patients with fatigue, pain and cramps. Int J Nurs Pract 19: 265-273. 